\title{
CLASSIFICATION OF FINITE-DIMENSIONAL UNIVERSAL PSEUDO-BOUNDARIES AND PSEUDO-INTERIORS
}

\author{
J. J. DIJKSTRA, J. vAN MILL AND J. MOGILSKI
}

\begin{abstract}
Let $n$ and $k$ be fixed integers such that $n \geq 1$ and $0 \leq k \leq$ $n$. Let $B_{k}^{n}$ and $s_{k}^{n}$ denote the $k$-dimensional universal pseudo-boundary and the $k$-dimensional universal pseudo-interior in $\mathbf{R}^{n}$, respectively. The aim of this paper is to prove that $B_{k}^{n}$ is homeomorphic to $B_{k}^{m}$ if and only if $s_{k}^{n}$ is homeomorphic to $s_{k}^{m}$ if and only if $n=m$ or $n, m \geq 2 k+1$.
\end{abstract}

\section{INTRODUCTION}

Let $n$ and $k$ be fixed integers such that $n \geq 1$ and $0 \leq k \leq n$. In addition, let $\mathscr{M}_{k}^{n}$ denote the collection of "tame" at most $k$-dimensional compacta in $\mathbf{R}^{n}$ (for precise definitions, see $\S 2$ ). In [10], Geoghegan and Summerhill proved that there exists an $\mathscr{M}_{k}^{n}$-absorber. This is the " $k$-dimensional universal pseudo-boundary in $\mathbf{R}^{n}$ " and we denote it by $B_{k}^{n}$. The $k$-dimensional universal pseudo-interior $s_{k}^{n}$ is the complement of $B_{n-k-1}^{n}$ in $\mathbf{R}^{n}$. The aim of this paper is to prove that $B_{k}^{n}$ is homeomorphic to $B_{k}^{m}$ if and only if $n=m$ or $n, m \geq 2 k+1$.

\section{Preliminaries: Part 1}

If $X$ is a space then $\mathscr{H}(X)$ denotes the group of autohomeomorphisms of $X$; let $1_{X}$ or 1 stand for the identity. Let $\mathscr{U}$ be a collection of subsets of $X$. We say that mappings $f, g: Y \rightarrow X$ are $\mathscr{U}$-close if for each $y \in Y$ with $f(y) \neq g(y)$ there is a $U \in \mathscr{U}$ containing both $f(y)$ and $g(y)$. An element $h \in \mathscr{H}(X)$ is called a $\mathscr{U}$-push if there is an isotopy $H: X \times I \rightarrow X$ that is limited by $\mathscr{U}$ and that satisfies $H_{0}=1$ and $H_{1}=h(H$ is limited by $\mathscr{U}$ means that every path $\left\{H_{t}(x): t \in I\right\}$ is either a singleton or is contained in some element of $\mathscr{U}$ ). If $A$ is a closed subset of $X$ and $\mathscr{U}$ an open cover of $X \backslash A$ then $\mathscr{U}$ is called a normal cover $\operatorname{rel} A$ if for each $a \in A$ and each neighbourhood $V$ of $a$ in $X$ there is a $W$ of $a$ such that $\operatorname{St}(W, \mathscr{U}) \subseteq V$, cf. $[11,1.4 .12]$. If the continuous map $f: X \backslash A \rightarrow X \backslash A$ is $\mathscr{U}$-close to 1 where $\mathscr{U}$ is normal rel $A$ then $f \cup 1_{A}$ is also continuous. Let $n$ and $k$ be fixed integers with the properties $n \geq 1$ and $0 \leq k \leq n$.

2.1. Definition. $\mathscr{M}_{k}^{n}$ consists of all compact subsets of $S$ of $\mathbf{R}^{n}$ that have the following property: if $P$ is a subpolyhedron of $\mathbf{R}^{n}$ (i.e. the underlying set of a simplicial complex in $\mathbf{R}^{n}$ ) with dimension at most $n-k-1$ and $\mathscr{U}$ is a

Received by the editors August 25, 1989 and, in revised form, May 12, 1990.

1980 Mathematics Subject Classification (1985 Revision). Primary 57N15, 57N20. 
collection of open subsets of $\mathbf{R}^{n}$ that covers $S \cap P$ then there exists a $\mathscr{U}$-push $h$ of $\mathbf{R}^{n}$ with $h(S) \cap P=\varnothing$. In addition, $\sigma \mathscr{M}_{k}^{n}$ is the collection of all countable unions of elements of $\mathscr{M}_{k}^{n}$.

Intuitively, $\mathscr{M}_{k}^{n}$ is the collection of "tame" at most $k$-dimensional compacta in $\mathbf{R}^{n}$. Note that if $P$ is an at most $k$-dimensional compact subpolyhedron of $\mathbf{R}^{n}$ then by a general position argument we find that $P \in \mathscr{M}_{k}^{n}$. Let $N_{k}^{n}$ be the $k$-dimensional Nöbeling space in $\mathbf{R}^{n}$, i.e. $N_{k}^{n}$ is the set of all points in $\mathbf{R}^{n}$ for which at most $k$ coordinates are rational. The collection $\mathscr{M}_{k}^{n}$ can be characterized as follows (see [7, Lemma 1.10]).

2.2. Lemma. $\mathscr{K}_{k}^{n}=\left\{f(S): f \in \mathscr{H}\left(\mathbf{R}^{n}\right)\right.$ and $S$ is a compactum in $\left.N_{k}^{n}\right\}$.

2.3. Definition. An element of $\sigma \mathscr{M}_{k}^{n}$ is called an $\mathscr{M}_{k}^{n}$-absorber if for every $S \in \mathscr{M}_{k}^{n}$ and every collection $\mathscr{U}$ of open subsets of $\mathbf{R}^{n}$ there is an $h \in \mathscr{H}\left(\mathbf{R}^{n}\right)$ that is $\mathscr{U}$-close to 1 while moreover $h(S \cap \bigcup \mathscr{U}) \subseteq A$.

Geoghegan and Summerhill have shown in [10] that there exists an $\mathscr{M}_{k}^{n}$ absorber. This is the $k$-dimensional universal pseudo-boundary in $\mathbf{R}^{n}$ and we denote it by $B_{k}^{n}$. The $k$-dimensional pseudo-interior $s_{k}^{n}$ is the complement of $B_{n-k-1}^{n}$ in $\mathbf{R}^{n}$.

We now sketch the construction of $B_{k}^{n}$. In essence, $B_{k}^{n}$ is a countable union of Menger compacta which are constructed by the "middle third" method. Define for $i=0,1,2, \ldots, K_{i}=\left\{(m+1 / 2) 3^{-i}: m\right.$ an integer $\}$ and let $\mathscr{P}$ be the group of homeomorphisms of $\mathbf{R}^{n}$ that correspond to coordinate permutations. We denote the open $\varepsilon$-ball with respect to the max metric in $\mathbf{R}^{n}$ by $U_{\varepsilon}$. Define for $m \in \mathbf{N}$ the following closed subsets of $\mathbf{R}^{n}$

$$
A_{m}=\mathbf{R}^{n} \backslash \bigcup_{i=0}^{\infty} \bigcup_{\alpha \in \mathscr{D}} \alpha\left(U_{\frac{1}{2} 3^{-i-m}}\left(K_{i}^{k+1} \times \mathbf{R}^{n-k-1}\right)\right) .
$$

Then the set $B_{k}^{n}=\bigcup_{m=1}^{\infty} A_{m}$ is an $\mathscr{M}_{k}^{n}$-absorber. For details, see Geoghegan and Summerhill [10] and Dijkstra [7].

We have the following important property for absorbers, see $[15,16]$.

2.4. Lemma. If $S$ is an element of $\sigma \mathscr{M}_{k}^{n}, \mathscr{U}$ a collection of open sets in $\mathbf{R}^{n}$ and $A$ is an $\mathscr{M}_{k}^{n}$-absorber then there exists an $h \in \mathscr{H}\left(\mathbf{R}^{n}\right), \mathscr{U}$-close to 1 , such that

$$
h(A \cap \bigcup \mathscr{U})=(S \cup A) \cap \bigcup \mathscr{U} .
$$

If in addition $S$ is itself an $\mathscr{M}_{k}^{n}$-absorber then we can replace (1) by

$$
h(A \cap \bigcup \mathscr{U})=S \cap \bigcup \mathscr{U} .
$$

Observe that this lemma states that absorbers are "unique," i.e. if $A$ and $B$ are $\mathscr{M}_{k}^{n}$-absorbers then there exists an $h \in \mathscr{H}\left(\mathbf{R}^{n}\right)$, which can be chosen arbitrarily close to 1 , such that $h(A)=B$.

We need the following thinness property.

2.5. Lemma. If $S \in \sigma \mathscr{M}_{k}^{n}$ and $R \in \sigma \mathscr{M}_{n-k-1}^{n}$ then there is an $h \in \mathscr{H}\left(\mathbf{R}^{n}\right)$, which can be chosen arbitrarily close to 1 , such that $h(S) \cap R=\varnothing$.

Proof. If $P$ is a compact subpolyhedron of $\mathbf{R}^{n}$ and $\mathscr{U}$ is an open covering of $\mathbf{R}^{n}$ then a $\mathscr{U}$-regular neighbourhood of $P$ is a regular neighbourhood $N$ of $P$ 
such that for any compactum $F$ in $\mathbf{R}^{n} \backslash P$ there is a $\mathscr{U}$-push $h$ of $\mathbf{R}^{n}$ with $h(F) \cap N=\varnothing$, cf. $[9, \S 3]$.

The absorbers $B_{k}^{n}$ and $B_{n-k-1}^{n}$ are constructed in [10] as countable unions of Menger compacta, standardly imbedded according to the "middle third" method. The procedure in [10, p. 153], representing the Menger compacta as intersections of regular neighbourhoods of polyhedra, shows that for every Menger compactum $M_{k}^{n}$ in $B_{k}^{n}$ and open covering $\mathscr{U}$ of $\mathbf{R}^{n}$ there is a $k$ dimensional subpolyhedron $P$ of $\mathbf{R}^{n}$ and a $\mathscr{U}$-regular neighbourhood $U$ of $P$ that contains $M_{k}^{n}$. Of course we have a similar statement for the Menger compacta $M_{n-k-1}^{n}$ that form $B_{n-k-1}^{n}$.

It is now easy to show that each such $M_{k}^{n}$ be pushed away from each such $M_{n-k-1}^{n}$. Let $\mathscr{U}$ be an open covering of $\mathbf{R}^{n}$. Select polyhedra $P$ and $Q$ in $\mathbf{R}^{n}$ with $\mathscr{U}$-regular neighbourhoods $U$ and $V$, respectively, such that $M_{k}^{n} \subseteq U$, $M_{n-k-1}^{n} \subseteq V, \operatorname{dim} P=k$ and $\operatorname{dim} Q=n-k-1$. By general position there is a $\mathscr{U}$-push $\alpha$ of $\mathbf{R}^{n}$ with the property $\alpha(P) \cap Q=\varnothing$. Since $V$ is a $\mathscr{U}$-regular neighbourhood there is a $\mathscr{U}$-push $\beta$ of $\mathbf{R}^{n}$ such that $\beta \circ \alpha(P) \cap V=\varnothing$. On the other hand

$$
P \cap \alpha^{-1} \circ \beta^{-1}(V)=\varnothing
$$

yields the existence of a $\mathscr{U}$-push $g$ with $U \cap \gamma \circ \alpha^{-1} \circ \beta^{-1}(V)=\varnothing$. So $\gamma \circ \alpha^{-1} \circ \beta^{-1}$ is a $\mathrm{St}^{2}(\mathscr{U})$-push of $\mathbf{R}^{n}$ with $M_{k}^{n} \cap \gamma \circ \alpha^{-1} \circ \beta^{-1}\left(M_{n-k-1}^{n}\right)=\varnothing$.

Let $\left\{A_{i}: i \in \mathbf{N}\right\}$ be the collection of Menger compacta that form $B_{k}^{n}$, let $\left\{B_{j}: j \in \mathbf{N}\right\}$ be the collection of Menger compacta that form $B_{n-k-1}^{n}$ and let $\left\{\left(i_{n}, j_{n}\right): n \in \mathbf{N}\right\}$ enumerate $\mathbf{N} \times \mathbf{N}$. By a standard induction procedure it is possible to construct a sequence $\left(h_{n}\right)_{n}$ in $\mathscr{H}\left(\mathbf{R}^{n}\right)$ such that $h_{n}$ pushes $h_{n-1} \circ \cdots \circ h_{1}\left(A_{i_{n}}\right)$ away from $B_{j_{n}}$ and keeping it disjoint from it in the limit, while moreover $h=\lim _{n \rightarrow \infty} h_{n} \circ \cdots \circ h_{1}$ is a homeomorphism of $\mathbf{R}^{n}$ which is arbitrarily close to the identity. Consequently, $h\left(B_{k}^{n}\right) \cap B_{n-k-1}=\varnothing$. Observing that by Lemma $2.4 B_{k}^{n}$ can absorb every element of $\sigma \mathscr{M}_{k}^{n}$ and $B_{n-k-1}^{n}$ can absorb every element of $\sigma \mathscr{M}_{n-k-1}^{n}$, we find the statement of Lemma 2.5 .

2.6. Lemma. If $S$ is a $G_{\delta}$-subset of $\mathbf{R}^{n}$ which contains $B_{k}^{n}$ then $\mathbf{R}^{n} \backslash S \in$ $\sigma \mathscr{M}_{n-k-1}^{n}$.

Proof. Observe that the complement of the Nöbeling space $N_{n-k-1}^{n}$ is a countable union of $k$-dimensional simplices in $\mathbf{R}^{n}$ and as such an element of $\sigma \mathscr{M}_{k}^{n}$. According to Lemma 2.4 we may therefore assume that $B_{k}^{n} \cup N_{n-k-1}^{n}=\mathbf{R}^{n}$, which means that $\mathbf{R}^{n} \backslash S \subseteq N_{n-k-1}^{n}$. So with Lemma 2.2 we may conclude that $\mathbb{R}^{n} \backslash S \in \sigma \mathscr{M}_{n-k-1}^{n}$.

Let $X$ and $Y$ be spaces and let $f: X \rightarrow Y$ be continuous. If $A \subseteq Y$ is closed then $(X, f)_{A}$ is the space with underlying set $\left(X \backslash f^{-1}(A)\right) \cup A$ and topology generated by the collection

$\left\{U \subseteq X \backslash f^{-1}(A): U\right.$ is open in $\left.X\right\} \cup\left\{f^{-1}(U \backslash A) \cup(U \cap A): U\right.$ is open in $\left.Y\right\}$.

Define $f_{A}: X \rightarrow(X, f)_{A}$ by

$$
f_{A}(x)= \begin{cases}x & \text { for } x \in X \backslash f^{-1}(A), \\ f(x) & \text { for } x \in f^{-1}(A) .\end{cases}
$$


It is easily seen that the function $f^{A}:(X, f)_{A} \rightarrow Y$ defined by

$$
f^{A} \circ f_{A}=f
$$

is continuous and has the following property:

For every $y \in A$ and every neighbourhood $V$ of $\left(f^{A}\right)^{-1}(y)$ in $(X, f)_{A}$ there exists a neighbourhood $U$ of $y$ in $Y$ with $\left(f^{A}\right)^{-1}(U) \subseteq V$.

We say that $f^{A}$ is closed over $A$. Formally, if $f: S \rightarrow T$ is continuous and $B \subseteq T$ is closed then $f$ is said to be closed over $B$ if for every $b \in B$ and every neighbourhood $V$ of $f^{-1}(b)$ in $S$ there exists a neighbourhood $U$ of $b$ in $T$ such that $f^{-1}(U) \subseteq V$.

Now let $A$ and $B$ be closed in $Y$ such that $A \subseteq B$. There is a unique map $f_{B}^{A}:(X, f)_{A} \rightarrow(X, f)_{B}$ such that $f_{B}=f_{B}^{A} \circ f_{A}$. Note that $f_{B}^{A}$ is closed over $A$.

Observe that $(Y \times Z, \pi)_{A}$, where $\pi: Y \times Z \rightarrow Y$ is the projection, is the cartesian product of $Y$ and $Z$ reduced over the closed set $A \subseteq Y$. This space will be denoted by $(Y \times Z)_{A}$, see [2, p. 25]. Finally observe that if $X$ and $Y$ are metrizable then for every closed set $A \subseteq Y$ also $(X, f)_{A}$ is metrizable, being homeomorphic to a subspace of $(Y \times X)_{A}$.

\section{Preliminaries: Part 2}

As usual, $\mathbf{R}^{\infty}$ denotes the countable infinite product of copies of $\mathbf{R}$. For each $k \in \mathbf{N} \cup\{0\}$ let $\mathscr{M}_{k}^{\infty}$ denote the collection of all at most $k$-dimensional compact subsets of $\mathbf{R}^{\infty}$. In addition, $\sigma \mathscr{M}_{k}^{\infty}$ is the collection of all countable unions of elements of $\mathscr{M}_{k}^{\infty}$.

3.1. Definition. An element $A$ of $\sigma \mathscr{M}_{k}^{\infty}$ is called an $\mathscr{M}_{k}^{\infty}$-absorber if for every $S \in \mathscr{M}_{k}^{\infty}$ and every collection $\mathscr{U}$ of open subsets of $\mathbf{R}^{n}$ there is an $h \in \mathscr{H}\left(\mathbb{R}^{\infty}\right)$ that is $\mathscr{U}$-close to 1 while, moreover, $h(S \cap \bigcup \mathscr{U}) \subseteq A$.

Dijkstra has shown in [7] that there exists an $\mathscr{M}_{k}^{\infty}$-absorber. We shall denote it by $B_{k}^{\infty}$. Again, we have the following absorption property, see [15, 16].

3.2. Lemma. If $S \in \sigma \mathscr{M}_{k}^{\infty}, \mathscr{U}$ a collection of open subsets of $\mathbf{R}^{\infty}$ and $A$ is an $\mathscr{M}_{k}^{\infty}$-absorber then there exists an $h \in \mathscr{H}\left(\mathbf{R}^{\infty}\right), \mathscr{U}$-close to 1 , such that

$$
h(A \cap \bigcup \mathscr{U})=(S \cup A) \cap \bigcup \mathscr{U} .
$$

If in addition $S$ is itself an $\mathscr{M}_{k}^{\infty}$-absorber then we can replace (1) by

$$
h(A \cap \bigcup \mathscr{U})=S \cap \bigcup \mathscr{U} .
$$

Again, observe that this lemma states that absorbers are "unique," i.e. if $A$ and $B$ are $\mathscr{M}_{k}^{\infty}$-absorbers then there exists an $h \in \mathscr{H}\left(\mathbf{R}^{\infty}\right)$, which can be chosen arbitrarily close to 1 , such that $h(A)=B$.

We shall use the spaces $B_{k}^{\infty}$ as tools in the process of proving that $B_{k}^{n}$ and $B_{k}^{m}$ are homeomorphic provided that $n, m \geq 2 k+1$.

The technique used in the proof of the following lemma is well known. Since the lemma is an essential step in our argumentation, we shall give its proof in full detail. 
3.3. Lemma. Let $A$ be an $\mathscr{M}_{k}^{\infty}$-absorber and let $G$ be a subset of $\mathbf{R}^{\infty}$ containing $A$. If $h \in \mathscr{H}(G)$ then $h(A)$ is an $\mathscr{M}_{k}^{\infty}$-absorber.

Proof. Standard homeomorphism extension results, see [2], imply that a subset $B$ of $\mathbf{R}^{\infty}$ is an $\mathscr{M}_{k}^{\infty}$-absorber if $B$ can be written as $B=\bigcup_{i=1}^{\infty} B_{i}$, where $B_{i} \in \mathscr{M}_{k}^{\infty}$ and $B_{i} \subseteq B_{i+1}$ for every $i$, while moreover the following property holds:

$\forall Z \in \mathscr{M}_{k}^{\infty} \forall n \in \mathbf{N} \forall \varepsilon>0$ :

$\exists m>n \exists$ imbedding $f: B_{n} \cup Z \rightarrow B_{m}$ such that $f \mid B_{n}=1$ and $d(f, 1)<\varepsilon$.

For details, see e.g. [6]. The tower $\left(B_{i}\right)_{i}$ is called a $k$-skeletoid. The $\mathscr{M}_{k}^{\infty}-$ absorber constructed in [7] is in fact a $k$-skeletoid.

Claim. Let $\left(B_{i}\right)_{i}$ be a $k$-skeletoid, let $G \subseteq \mathbf{R}^{\infty}$ such that $\bigcup_{i=1}^{\infty} B_{i} \subseteq G$ and let $h \in \mathscr{H}(G)$. Then $\left(h\left(B_{i}\right)\right)_{i}$ is a $k$-skeletoid.

First observe that $h\left(B_{i}\right) \in \mathscr{M}_{k}^{\infty}$ for every $i$. Now choose an arbitrary $Z \in$ $\mathscr{M}_{k}^{\infty}, n \in \mathbf{N}$ and $\varepsilon>0$. Since $\bigcup_{i=1}^{\infty} B_{i}$ is an $\mathscr{M}_{k}^{\infty}$-absorber, there exists an $\alpha \in \mathscr{H}\left(\mathbf{R}^{\infty}\right)$ such that

$$
d(\alpha, 1)<\frac{1}{2} \varepsilon, \quad \alpha \mid h\left(B_{n}\right)=1 \quad \text { and } \quad \alpha\left(Z \backslash h\left(B_{n}\right)\right) \subseteq \bigcup_{i=1}^{\infty} B_{i} .
$$

Observe that $\alpha(Z) \subseteq G$ and consider $h^{-1} \alpha(Z)$. By compactness of this set there clearly exists a $\delta>0$ such that for every $x \in h^{-1} \alpha(Z)$ and $y \in G$ with $d(x, y)<\delta$ we have $d(h(x), h(y))<\frac{1}{2} \varepsilon$. There are $m \in \mathbf{N}$ and $\beta \in \mathscr{H}\left(\mathbf{R}^{\infty}\right)$ such that

$$
d(\beta, 1)<\delta, \quad \beta \mid B_{n}=1 \quad \text { and } \quad \beta \circ h^{-1} \circ \alpha(Z) \subseteq B_{m} .
$$

Now define $\gamma: h\left(B_{n}\right) \cup Z \rightarrow \mathbf{R}^{\infty}$ by

$$
\gamma(x)=h \circ \beta \circ h^{-1} \circ \alpha(x) .
$$

Observe that $\gamma$ is a well-defined imbedding, that $\gamma \mid h\left(B_{n}\right)=1$ and that $\gamma(Z) \subseteq$ $h\left(B_{m}\right)$. We shall prove that $d(\gamma, 1)<\varepsilon$. To this end, take an arbitrary $x \in \bar{Z}$ and observe that $d\left(h^{-1} \circ \alpha(x), \beta \circ h^{-1} \circ \alpha(x)\right)<\delta$. Since $h^{-1} \circ \alpha(x) \in h^{-1} \circ \alpha(Z)$ this implies that

$$
d(\alpha(x), \gamma(x))=d\left(h \circ h^{-1} \circ \alpha(x), h \circ \beta \circ h^{-1} \circ \alpha(x)\right)<\frac{1}{2} \varepsilon .
$$

Consequently, since $d(\alpha, 1)<\frac{1}{2} \varepsilon$,

$$
d(x, \gamma(x)) \leq d(x, \alpha(x))+d(\alpha(x), \gamma(x))<\frac{1}{2} \varepsilon+\frac{1}{2} \varepsilon=\varepsilon .
$$

This completes the proof of the claim.

Now let $A$ and $G$ be as in the lemma. Since by [7] $k$-skeletoids exist, by the claim and Lemma 3.2, $A$ is a $k$-skeletoid. Again by the claim, $h(A)$ is a $k$-skeletoid, hence an $\mathscr{K}_{k}^{\infty}$-absorber.

Remark. The above lemma is false if we replace $\mathbf{R}^{\infty}$ by $Q$, where $Q$ denotes the Hilbert cube. To see this, for every $i \in \mathbb{N}$ let $E_{i} \subseteq Q$ be a Z-set Cantor set such that $\bigcup_{i=1}^{\infty} E_{i}$ is dense in $Q$. In [5] it was shown that $E=\cup_{i=1}^{\infty} E_{i}$ is an absorber for the collection of all zero-dimensional $Z$-sets in $Q$. Let $K \subseteq Q$ be a wild Cantor set [17]. Then $E \cup K$ is zero-dimensional and therefore is contained in a zero-dimensional $G_{\delta}$-subset $P \subseteq Q$. Observe that $P$ is homeomorphic to 
the space of irrational numbers and that both $E$ and $E \cup K$ are homeomorphic to the product of the rational numbers and the Cantor Set. By [8] there is a homeomorphism $f: P \rightarrow P$ mapping $E$ onto $E \cup K$. But $E \cup K$ is not an absorber for the collection of all zero-dimensional $Z$-sets in $Q$ since $K$ is not a $Z$-set.

3.4. Lemma. (a) Let $k \in \mathbf{N} \cup\{0\}$ and let $m \in \mathbf{N}$ be such that $2 k+1 \leq m$. Then for every $\mathscr{M}_{k}^{m}$-absorber $X$ in $\mathbf{R}^{m}$ there exists an $\mathscr{M}_{k}^{\infty}$-absorber $Y$ in $\mathbf{R}^{m} \times \mathbf{R}^{\infty}$ such that $\pi(Y)=X$, where $\pi: \quad \mathbf{R}^{m} \times \mathbf{R}^{\infty} \rightarrow \mathbf{R}^{m}$ is the projection.

(b) If $X$ is an $\mathscr{M}_{k}^{\infty}$-absorber in $\mathbf{R}^{\infty}$ then there exists an $\mathscr{M}_{k}^{\infty}$-absorber $Y$ in $\mathbf{R}^{\infty} \times \mathbf{R}$ such that $\pi(Y)=X$, where $\pi: \mathbf{R}^{\infty} \times \mathbf{R} \rightarrow \mathbf{R}^{\infty}$ is the projection.

Proof. (a) Let $Z$ be an $\mathscr{M}_{k}^{\infty}$-absorber in $\mathbf{R}^{m} \times \mathbf{R}^{\infty}$. Write $Z=\bigcup_{i=1}^{\infty} Z_{i}$ with $Z_{i} \in \mathscr{M}_{k}^{\infty}$ and $Z_{i} \subseteq Z_{i+1}$ for every $i$. Since $\operatorname{dim}\left(Z_{1}\right) \leq k$ and $2 k+1 \leq m$, the function $f_{1}=\pi \mid Z_{1}: Z_{1} \rightarrow \mathbf{R}^{m}$ is approximable by an imbedding $\bar{f}_{1}: Z_{1} \rightarrow N_{k}^{m}$ $[11, \S 4.4]$. Put

$$
S_{1}=\left\{\left(\bar{f}_{1}(x, y), y\right):(x, y) \in Z_{1}\right\}
$$

and define $g_{1}: Z_{1} \rightarrow S_{1}$ by $g_{1}(x, y)=\left(\bar{f}_{1}(x, y), y\right)$. Then $g_{1}$ is a homeomorphism which can be extended to a homeomorphism $\bar{g}_{1} \in \mathscr{H}\left(\mathbf{R}^{m} \times \mathbf{R}^{\infty}\right)$ [2]. Observe that $\pi$ restricted to $\bar{g}_{1}\left(Z_{1}\right)$ is an imbedding into $N_{k}^{m}$.

The function $f_{2}=\pi \mid \bar{g}_{1}\left(Z_{2}\right): \bar{g}_{1}\left(Z_{2}\right) \rightarrow \mathbf{R}^{m}$ is approximable by an imbedding $\bar{f}_{2}: \bar{g}_{1}\left(Z_{2}\right) \rightarrow N_{k}^{m}$ such that $\bar{f}_{2}\left|\bar{g}_{1}\left(Z_{1}\right)=\pi\right| \bar{g}_{1}\left(Z_{1}\right)$. Arguing as above yields a homeomorphism $\bar{g}_{2} \in \mathscr{H}\left(\mathbf{R}^{m} \times \mathbf{R}^{\infty}\right)$ having the following properties:

(1) $\bar{g}_{2}$ is as close to the identity as we please,

(2) $\bar{g}_{2} \mid \bar{g}_{1}\left(Z_{1}\right)=1$,

(3) $\pi$ restricted to $\bar{g}_{2}\left(Z_{2}\right)$ is an imbedding into $N_{k}^{m}$.

Continuation of this process yields the existence of a sequence $\left(\bar{g}_{i}\right)_{i}$ in $\mathscr{H}\left(\mathbf{R}^{m} \times \mathbf{R}^{\infty}\right)$ having the following properties:

(4) $g=\lim _{i \rightarrow \infty} \bar{g}_{i} \circ \cdots \circ \bar{g}_{1} \in \mathscr{H}\left(\mathbf{R}^{m} \times \mathbf{R}^{\infty}\right)$,

(5) for every $i \in \mathbf{N}$ we have that $\pi \mid g\left(Z_{i}\right): g\left(Z_{i}\right) \rightarrow \mathbf{R}^{m}$ is an imbedding into $N_{k}^{m}$.

From Lemmas 2.2 and 2.3 we may therefore conclude that without loss of generality $\pi(Z) \in \sigma \mathscr{M}_{k}^{m}$. By Lemmas 2.4 and 3.3 it now follows that without loss of generality, $\pi(Z) \subseteq X$. Let $\mathbf{0}$ be the point in $\mathbf{R}^{\infty}$ having all coordinates equal to 0 . By Lemma 3.2, $Y=Z \cup(X \times\{\mathbf{0}\})$ is an $\mathscr{M}_{k}^{\infty}$-absorber. Since $Y$ clearly projects onto $X$, it is as required.

The proof of (b) is similar to the proof of (a).

A closed subset $A$ of a space $X$ is called $Z$-set if for every open cover $\mathscr{U}$ of $X$ there is a map $f: X \rightarrow X \backslash A$ which is $\mathscr{U}$-close to the identity.

3.5. Theorem. Let $X$ be an $\mathscr{M}_{k}^{\infty}$-absorber, let $\mathscr{U}$ be a collection of open subsets of $\mathbf{R}^{\infty}$ and let $A$ and $B$ be $Z$-sets in $X$ that the closures of $A$ and $B$ in $\mathbf{R}^{\infty}$ are $Z$-sets in $\mathbf{R}^{\infty}$. If $h: A \rightarrow B$ is a homeomorphism which is homotopic in $\mathbf{R}^{\infty}$ to the inclusion $A \rightarrow X$ by a homotopy that is limited by $\mathscr{U}$ then for every open cover $\mathscr{V}$ of $\bigcup \mathscr{U}$ there exists a homeomorphism $H: X \rightarrow X$ such that

(1) $H \mid A=h$.

(2) $H$ is $\operatorname{St}(\operatorname{St}(\mathscr{U}), \mathscr{V})$-close to the identity on $X$. 
Proof. Let $O=\bigcup \mathscr{U}$. Observe that $h$ restricts the identity on $A \backslash O$. This implies that there exists an open cover $\mathscr{W}$ of $O$ such that

(3) $\mathscr{W}$ refines $\mathscr{U}$,

(4) $h$ is homotopic in $\mathbf{R}^{\infty}$ to the inclusion $A \rightarrow X$ by a homotopy that is limited by $\mathscr{W}$,

(5) $\mathscr{W}$ is normal rel $\mathbf{R}^{\infty} \backslash O$.

By arguing precisely as in Chapman $[4, \S 11]$ we can construct $G_{\delta}$-sets $\vec{A} \subseteq \bar{A}$ and $\vec{B} \subseteq \bar{B}$ such that $h$ can be extended to a homeomorphism $\vec{h}: \vec{A} \rightarrow \vec{B}$ such that $\vec{h}$ and the inclusion $\vec{A} \rightarrow \mathbf{R}^{\infty}$ are homotopic in $\mathbf{R}^{\infty}$ by a homotopy that is limited by $\operatorname{St}(\mathscr{W})$. Since $\bar{A}$ and $\bar{B}$ are $Z$-sets in $\mathbf{R}^{\infty}$ it follows that $(\bar{A} \cup \bar{B}) \backslash(\vec{A} \cup \vec{B})$ is a countable union of $Z$-sets in $\mathbf{R}^{\infty}$. Put

$$
W=\mathbf{R}^{\infty} \backslash((\bar{A} \cup \bar{B}) \backslash(\vec{A} \cup \vec{B})) \quad \text { and } \quad V=O \backslash((\bar{A} \cup \bar{B}) \backslash(\vec{A} \cup \vec{B})),
$$

respectively. By [1], $\mathbf{R}^{\infty}$ and $W$ are homeomorphic. Observe that $V$ is open in $W$. It is a triviality to verify that $X \cap W$ is a $k$-skeletoid in $W$, see e.g. the proof of Lemma 3.3. Since $(\overrightarrow{A \backslash} \backslash \vec{A}) \cup(\vec{B} \backslash \vec{B})$ is, are remarked above, a countable union of $Z$-sets in $\mathbf{R}^{\infty}$, it also follows that $\vec{h}$ and the inclusion $\vec{A} \rightarrow W$ are homotopic by a homotopy that is limited by $\operatorname{St}(\mathscr{W} \cap V)$. Since $\vec{A}$ and $\vec{B}$ are $Z$-sets in $W, \vec{h}$ extends to a homeomorphism $\bar{h}: W \rightarrow W$ which is $\operatorname{St}(\mathscr{W} \cap V)$-close to the identity on $W$ [2]. Since $\bar{h}(X)$ is a $k$-skeletoid in $W$ there exists a homeomorphism $g: W \rightarrow W$ which is $\mathscr{V} \cap V$-close to the identity and which moreover has the properties $g \circ \bar{h}(X)=X$ and $g \mid \vec{B} \cup(W \backslash V)=1$. Let $H=g \circ \bar{h} \mid X$. Then $H$ has the required properties.

3.6. Corollary. Let $X$ be an $\mathscr{M}_{k}^{\infty}$-absorber. If $\mathscr{U}$ is a collection of open subsets of $X$ then there exists an open cover $\mathscr{V}$ of $\bigcup \mathscr{U}$ such that whenever we have a pair of imbeddings $\alpha_{1}$ and $\alpha_{2}$ of a compactum into $X$ that are $\mathscr{V}$-close then there is a homeomorphism $h$ of $X$ that is $\mathscr{U}$-close to 1 with the property $h \circ \alpha_{1}=\alpha_{2}$.

\section{Positive Results}

In this section we shall present our main result, the proof of which is inspired by Toruńczyk [13, 14], Mogilski [12] and Bestvina and Mogilski [3].

Let $f: Y \rightarrow X$ be a continuous surjection. Then $f$ is called a near-homeomorphism provided that for every open cover $\mathscr{U}$ of $X$ there is a homeomorphism $h: Y \rightarrow X$ which is $\mathscr{U}$-close to $f$. We shall need the following version of Bing's Shrinking Criterion due to Torunczyk [14].

4.1. Theorem. Let $f: Y \rightarrow X$ be a continuous surjection, let $B \subseteq X$ be closed and let $K \subseteq Y$ be closed such that $f(K)=B$. Suppose that for every open cover $\mathscr{U}$ of $X$, for every open cover $\mathscr{V}$ of $Y$ and for every neighbourhood $U$ of $B$ there are a homeomorphism $h: Y \rightarrow Y$ and an open cover $\mathscr{W}$ of $X$ such that

(1) $f$ and $f \circ h$ are $\mathscr{U}$-close,

(2) $h(x)=x$ if $x \in K \cup f^{-1}(X \backslash U)$,

(3) for every $W \in \mathscr{W}$ there is a $V \in \mathscr{V}$ such that $h\left(f^{-1}(W)\right) \subseteq V$.

Then $f$ is a near-homeomorphism. 
Remark. Observe that the map $f$ in the above theorem has the property that $f \mid K: K \rightarrow B$ is a homeomorphism. In addition, all "shrinking" homeomorphisms restricted to $K$ are the identity. This implies that the homeomorphisms that are going to approximate $f$ all have the property that they agree with $f$ on $K$.

Let $C=\left(\mathbf{R}^{\infty} \times[0,1]\right)_{\{1\}}$ be the cone over $\mathbf{R}^{\infty}$. It is well known that $C$ is homeomorphic to $\mathbf{R}^{\infty}$. In addition, let $k \in \mathbf{N} \cup\{0\}$ and let $m \in \mathbf{N}$ such that $2 k+1 \leq m$. Finally, let $X \subset \mathbf{R}^{m}$ be an $\mathscr{M}_{k}^{m}$-absorber.

By Lemma 3.4 (a) there exists an $\mathscr{M}_{k}^{\infty}$-absorber $X_{1}$ in $\mathbf{R}^{m} \times \mathbf{R}^{\infty}$ such that $X_{1} \subseteq X \times \mathbf{R}^{\infty}$ and $X_{1} \cap\left(\{x\} \times \mathbf{R}^{\infty}\right) \neq \varnothing$ for every $x \in X$.

We shall now derive a technical lemma that will be important later. Let $A \in \mathscr{M}_{m-k-1}^{m}$ and let $U$ be an open neighbourhood of $A$ in $\mathbf{R}^{m}$. Pick an arbitrary (admissible) metric $d_{m}$ on $U$ and an arbitrary metric $d_{\infty}$ on $\mathbf{R}^{\infty}$. On $U \times \mathbf{R}^{\infty}$ we use the metric

$$
d((x, y),(s, t))=\max \left\{d_{m}(x, s), d_{\infty}(y, t)\right\} .
$$

4.2. Lemma. There is a closed imbedding $f: X_{1} \rightarrow X_{1}$ with the following properties:

(1) $d\left(f\left(X_{1}\right), A \times \mathbf{R}^{\infty}\right)>0$,

(2) $f$ is supported on $U \times \mathbf{R}^{\infty}$,

(3) $f \mid\left(U \times \mathbf{R}^{\infty}\right)$ is arbitrarily close to 1 (with respect to the metric $d$ ).

Proof. Since $A \in \mathscr{M}_{m-k-1}^{m}$ there is a regular $\varepsilon$-neighbourhood $N \subseteq U$ of an $(m-k-1)$-dimensional polyhedron $P$ such that $A \subseteq \operatorname{Int}(N)$, with $\varepsilon$ arbitrarily small [10]. Choose $\alpha \in \mathscr{H}\left(\mathbf{R}^{m}\right)$, close to 1 with respect to the metric $d_{m}$, such that $\alpha(X) \cap P=\varnothing$. There is a function $p: \partial N \times[0,1] \rightarrow N$ such that $p: \partial N \times[0,1) \rightarrow N \backslash P$ is a homeomorphism, $p(\partial N \times\{1\})=P$, and

$$
\operatorname{diam} p(\{x\} \times[0,1])<\varepsilon \text { for every } x \in \partial N .
$$

Pick $\delta>0$ such that $p(\partial N \times[0, \delta]) \cap A=\varnothing$ and define $N_{\delta}=p(\partial N \times[\delta, 1])$. Let $\beta: \mathbf{R}^{m} \backslash P \rightarrow \mathbf{R}^{m} \backslash N_{\delta}$ be a homeomorphism, defined in the obvious way, which is supported on $\operatorname{Int}(N)$. Notice that $\partial N_{\delta} \times \mathbf{R}^{\infty}$ is a $Z$-set in the Hilbert space manifold $M=\left(\mathbf{R}^{m} \backslash \operatorname{Int}\left(N_{\delta}\right)\right) \times \mathbf{R}^{\infty}$, and consequently there exists a homeomorphism $\gamma: M \backslash\left(\partial N_{\delta} \times \mathbf{R}^{\infty}\right) \rightarrow M$ which is close to 1 with respect to the metric $d$, [1]. Notice that $\vec{X}_{1}=\gamma \circ\left((\beta \circ \alpha) \times 1_{\mathbf{R}} \infty\right)\left(X_{1}\right)$ and $X_{1} \cap M$ are both absorbers for the $\leq k$-dimensional compacta in $M$ (self-explanatory). So there exists a $\xi \in \mathscr{H}(M)$ such that $\xi\left(\vec{X}_{1}\right)=X_{1} \cap M$ while moreover $\xi$ and 1 are close with respect to the metric $d$, Lemma 3.2. Now define

$$
f=\xi \circ \gamma \circ\left((\beta \circ \alpha) \times 1_{\mathbf{R}^{\infty}}\right) \mid X_{1} .
$$

Since $M$ is closed, $f$ is as required.

By Lemma 3.4(b) there exists an $\mathscr{M}_{k}^{\infty}$-absorber $X_{2}$ in $\mathbf{R}^{m} \times \mathbf{R}^{\infty} \times(0,1)$ such that $X_{2} \subseteq X_{1} \times(0,1)$ and $X_{2} \cap(\{x\} \times(0,1)) \neq \varnothing$ for every $x \in X_{1}$. Let $\xi: \mathbf{R}^{\infty} \times[0,1] \rightarrow C$ be the "projection." (See Figure 1.) In addition, let $\eta: \mathbf{R}^{m} \times \mathbf{R}^{\infty} \times[0,1] \rightarrow \mathbf{R}^{m} \times C$ be the map $1_{\mathbf{R}^{m}} \times \xi$. (See Figure 2.)

Since $\eta\left(\mathbf{R}^{m} \times \mathbf{R}^{\infty} \times\{0,1\}\right)$ is clearly a $Z$-set in $\mathbf{R}^{m} \times C$, it easily follows that $\eta\left(X_{2}\right)$ is an $\mathscr{M}_{k}^{\infty}$-absorber in $\mathbf{R}^{m} \times C$. Consequently,

$$
Y=\eta\left(X_{2} \cup\left(X_{1} \times\{0,1\}\right)\right)
$$




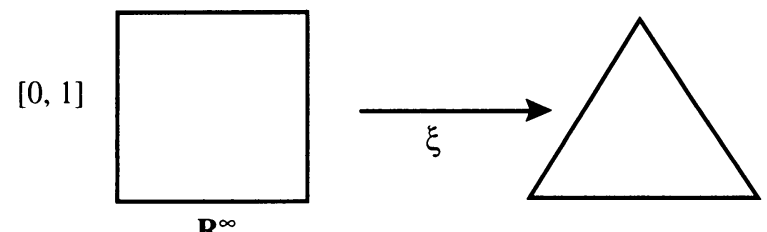

FIGURE 1

$[0,1]$
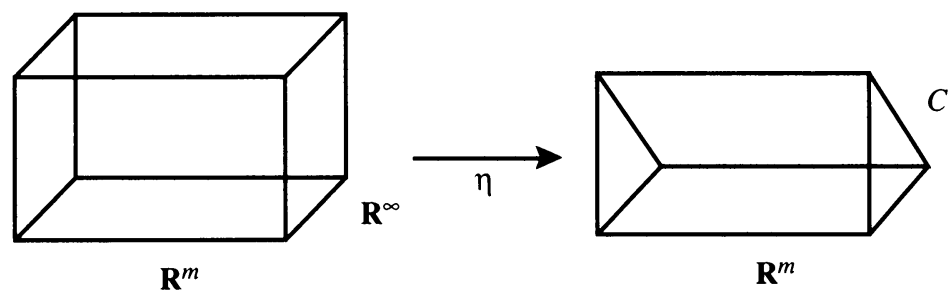

$\mathbf{R}^{m}$

FIGURE 2

is also an $\mathscr{M}_{k}^{\infty}$-absorber; simply observe that $\eta\left(X_{1} \times\{0\}\right)$ is homeomorhic to $X_{1}$ and $\eta\left(X_{1} \times\{1\}\right)$ is homeomorphic to $X$ and apply Lemma 3.2. Let $\pi: \mathbf{R}^{m} \times C \rightarrow \mathbf{R}^{m}$ be the projection and observe that $\pi(Y)=X$.

We shall prove

\subsection{Proposition. $f=\pi \mid Y: Y \rightarrow X$ is a near-homeomorphism.}

It will be convenient to introduce some notation. If $A$ is a compact subset of $X$ then

$$
\pi_{A}: \mathbf{R}^{m} \times C \rightarrow\left(\mathbf{R}^{m} \times C\right)_{A} \quad \text { and } \quad \pi^{A}:\left(\mathbf{R}^{m} \times C\right)_{A} \rightarrow \mathbf{R}^{m}
$$

have been defined in $\S 2$. Let $Y_{A}$ stand for $\pi_{A}(Y)$ and put $f_{A}=\pi_{A} \mid Y$ and $f^{A}=\pi^{A} \mid Y_{A}$. Now, let $B$ be a compact subset of $X$ that contains $A$. Then $\pi_{B}^{A}:\left(\mathbf{R}^{m} \times C\right)_{A} \rightarrow\left(\mathbf{R}^{m} \times C\right)_{B}$ is well defined and we may put $f_{B}^{A}=\pi_{B}^{A} \mid Y_{A}: Y_{A} \rightarrow$ $Y_{B}$.

If $S$ is a subset of $\mathbf{R}^{m}$ then $S^{*}$ denotes the homeomorphic subset $\eta(S \times$ $\left.\mathbf{R}^{\infty} \times\{1\}\right)$ of $\mathbf{R}^{m} \times C$. If $S$ is a subset of $\mathbf{R}^{m} \times \mathbf{R}^{\infty}$ then $S_{*}$ denotes the homeomorphic subset $\eta(S \times\{0\})$ of $\mathbf{R}^{m} \times C$.

The most important step in the proof of Proposition 4.3 is the following:

4.4. Lemma. Let $A$ and $B$ be compact subsets of $X$ such that $A \subseteq B$. Moreover, let $K$ be a compact subset of $Y_{A}$ such that $f^{A} \mid K$ is a homeomorphism from $K$ onto $B$. If $\mathscr{U}$ is an open cover of $X \backslash A$ then there is a homeomorphism $h: Y_{A} \rightarrow Y_{B}$ such that $f^{B} \circ h$ is $\mathscr{U}$-close to $f^{A}$ and $h\left|K=f_{B}^{A}\right| K$.

From the proof of Lemma 4.4 it will follow that we can choose the homeomorphism $h$ to be "supported" on an arbitrarily small neighbourhood of $B$. Therefore, by putting $A=\varnothing$ and $K=B^{*}$ we find

4.5. Corollary. If $B \subseteq X$ is compact then $f_{B}: Y \rightarrow Y_{B}$ is a near-homeomorphism.

Remark. At first glance this is surprising because it seems that the maps involved are rather arbitrary. However, because of the special nature of the absorbers, certain homotopy conditions are automatically satisfied. We illustrate this by verifying the following: 
Fact. Let $p: \mathbf{R}^{m} \rightarrow \mathbf{R}^{n}$ be the projection, where $m>n$. Then if $Y \subseteq \mathbf{R}^{m}$ is an $\mathscr{M}_{k}^{m}$-absorber, then $p \mid Y$ is a $U V^{k}$-map (i.e. given $x \in \mathbf{R}^{n}, j \leq \bar{k}$ and $a$ neighbourhood $U$ of $x$, there is a neighbourhood $V \subseteq U$ of $x$ such that each $g: \partial I^{j} \rightarrow p^{-1}(V) \cap Y$ extends to a map $\left.g: I^{j} \rightarrow p^{-1}(U) \cap Y\right)$.

Proof. Let $x \in \mathbf{R}^{n}, j \leq k$ and $x \in U \subseteq \mathbf{R}^{n}$, where $U$ is open. We can choose a neighbourhood $V$ of $x$ in $\mathbf{R}^{n}$, such that $V \subseteq U$ while moreover each map $g: \partial I^{j} \rightarrow p^{-1}(V) \cap Y$ extends to a map $g: I^{j} \rightarrow p^{-1}(U)$. By the absorption property of $Y$ we can adjust $g$ such that $g\left(I^{j}\right) \subseteq p^{-1}(U) \cap Y$.

Proof of Lemma 4.4. Obviously, our aim is to apply Theorem 4.1 to $f_{B}^{A}: Y_{A} \rightarrow$ $Y_{B}$. In the proof we shall work in the spaces $Y$ and $X$ rather than in $Y_{A}$ and $Y_{B}$. Because $f^{B}: Y_{B} \rightarrow X$ is closed over $B$ it suffices to consider an arbitrary open cover $\mathscr{U}$ of $X \backslash A$ together with an open neighbourhood $U$ of $B$ in $X$. Furthermore, instead of finding a homeomorphism of $Y_{A}$ we shall construct a homeomorphism $g$ of $Y$ with the property that there is a homeomorphism $\tilde{g}$ of $Y_{A}$ such that $\tilde{g} \circ f_{A}=f_{A} \circ g$. This can be realized by letting $g$ be supported on a set $f^{-1}(Q)$ where $Q$ is a closed set in $\mathbf{R}^{m}$ that is disjoint from $A$.

The homeomorphism $g$ will be a composite of six homeomorphisms, namely:

$$
g=\alpha^{-1} \circ \gamma \circ \bar{h}_{2} \circ \bar{h}_{1} \circ \beta \circ \alpha \mid Y \text {. }
$$

The map $\alpha$ moves the bulk of the set $K \backslash A$ to the top of the cone $C$, whereas $\beta$ is an adjustment homeomorphism that pushes $\alpha(Y)$ back to $Y$. The functions $\bar{h}_{1}$ and $\bar{h}_{2}$ do the actual shrinking: the fibres of $B$ are compressed into a small neighbourhood of the top of the cone. The map $\gamma$ pushes $\bar{h}_{2} \circ \bar{h}_{1}(Y)$ onto $\alpha(Y)$. Finally, $\alpha^{-1}$ moves $K$ back to its original place.

Construction of $\alpha$. Let $\mathscr{V}$ be an open covering of $Y_{A}$ and let $\mathscr{U}$ and $U$ be as above. We assume that the elements of $\mathscr{V}$ respectively $\mathscr{U} \cup\{U\}$ have been extended to open subsets of $\left(\mathbf{R}^{m} \times C\right)_{A}$ respectively $\mathbf{R}^{m}$. Since $\pi^{A}$ : $\left(\mathbf{R}^{m} \times C\right)_{A} \rightarrow \mathbf{R}^{m}$ is closed over $A$ we can find an open covering $\mathscr{V}_{1}$ of $A$ in $U$ such that $\pi^{-1}\left(\mathscr{V}_{1}\right)$ refines $\pi_{A}^{-1}(\mathscr{V})$. Let $O_{1}$ and $O_{2}$ be open subsets in $\mathbf{R}^{m}$ such that $A \subseteq O_{1} \subseteq \bar{O}_{1} \subseteq O_{2} \subseteq \bar{O}_{2} \subseteq \cup \mathscr{V}_{1}$. Define $B_{i}=B \backslash O_{i}$ and $K_{i}=K \backslash\left(\pi^{A}\right)^{-1}\left(O_{i}\right)$ for $i=1,2$. Put $U_{1}=U \backslash A$ and $U_{2}=U \backslash \bar{O}_{1}$. Since $C$ is homeomorphic to Hilbert space we may assume that it is endowed with a vector space structure in which the vertex of $C$ corresponds to the zero vector 0. Select a continuous $\phi: \mathbf{R}^{m} \rightarrow C$ such that

$$
\phi(b)=\left(f^{A} \mid K\right)^{-1}(b) \text { for } b \in B_{1}
$$

and $\phi(x)=\mathbf{0}$ for $x \notin U_{1}$. Let $\alpha$ be a homeomorphism of $\mathbf{R}^{m} \times C$ defined by

$$
\alpha(x, y)=(x, y-\phi(x)) .
$$

Observe that $\alpha$ is supported on $U_{1} \times C$ and that $\alpha\left(K_{1}\right)=B_{1}^{*}$.

Construction of $\bar{h}_{1}$. Use the compactness of $B_{1}^{*}$ to find an open covering $\mathscr{W}_{1}$ of $B_{1}$ in $U_{1}$ and an $\varepsilon>0$ such that

$$
\left\{\eta\left(W \times \mathbf{R}^{\infty} \times(1-\varepsilon, 1]\right): W \in \operatorname{St}^{3}\left(\mathscr{W}_{1}\right)\right\}
$$

refines both $\alpha\left(\pi_{A}^{-1}(\mathscr{V})\right)$ and $\pi^{-1}(\mathscr{U})$ and moreover such that

$$
\left\{\operatorname{St}^{3}\left(W, \mathscr{W}_{1}\right): W \in \mathscr{W}_{1} \text { and } W \cap \bar{O}_{1} \neq \varnothing\right\} \text { refines } \mathscr{V}_{1} \text {. }
$$


Let $\mathscr{W}$ be an open cover of $B_{2}$ defined by

$$
\mathscr{W}=\left\{W \cap U_{2}: W \in \mathscr{W}_{1}\right\} .
$$

According to Lemma 4.2 there is a closed imbedding $v: X_{1 *} \rightarrow X_{1 *}$ and a closed neighbourhood $P$ of $B_{2}$ in $\bigcup \mathscr{W}$ with the range of $v$ disjoint from $P \times C$. Note that both domain and range of $v$ are $Z$-sets in $Y$. We may assume that $v$ is so close to the identity that the extension homeomorphism $h_{1}: Y \rightarrow Y$ for the map $v^{-1}$ (which exists by Theorem 3.5) has the properties that $f \circ h_{1}$ is $\mathscr{W}$-close to $f$ and that $h_{1}$ fixes $B_{1}^{*}$. We may also assume that $h_{1}$ can be extended by the identity on $\left(\mathbf{R}^{m} \backslash \cup \mathscr{W}\right) \times C$ to a homeomorphism $\bar{h}_{1}$ of $Y \cup\left(\left(\mathbf{R}^{m} \backslash \cup \mathscr{W}\right) \times C\right)$.

Construction of $\bar{h}_{2}$. We have $\bar{h}_{1}\left(f^{-1}(P)\right)$ is disjoint from $X_{1 *}$, so we can insert between these closed subsets of $Y$ the "graph"

$$
\left\{\eta(z, \delta(z)): z \in X_{1}\right\}
$$

of a map $\delta: X_{1} \rightarrow(0,1)$ with the property $\delta(x, y)=1-\varepsilon \backslash 2$ if $x \notin \bigcup \mathscr{W}$. Then use the map $\delta$ to get a homeomorphism $h_{2}$ of $\eta\left(X_{1} \times[0,1]\right)$ that moves points along the $[0,1]$-axis of $C$ and pushes the "graph" $\left\{\eta(z, \delta(z)): z \in X_{1}\right\}$ onto the "plane" $\eta\left(X_{1} \times\{1-\varepsilon / 2\}\right)$. Since $\delta(x, y)=1-\varepsilon / 2$ for $x \notin \bigcup \mathscr{W}$ we may assume that $h_{2}$ is supported on $\pi^{-1}(\bigcup \mathscr{W})$. The map $h_{2}$ obviously fixes $X^{*}$. Again put $\bar{h}_{2}=h_{2} \cup 1_{\left(\mathbf{R}^{m} \backslash \bigcup \mathscr{W}\right) \times C}$.

Construction of $\beta$. We want $\beta$ to push $\alpha(Y) \cap \pi^{-1}(\bigcup \mathscr{W})$ back to $Y$. The set $\alpha(Y)$ is obviously an $\mathscr{M}_{k}^{\infty}$-absorber in $\mathbf{R}^{m} \times C$. Let $\mathscr{W}^{\prime}$ be an open covering of $\bigcup \mathscr{W}$ such that $\operatorname{St}^{2}\left(B_{2}, \mathscr{W}^{\prime}\right) \subseteq P$ and $\mathscr{W}^{\prime}$ refines $\mathscr{W}$. According to Lemma 3.2 there is a homeomorphism $\beta$ of $\mathbf{R}^{m} \times C$ that is $\pi^{-1}\left(\mathscr{W}^{\prime}\right)$-close to 1 such that

$$
\beta\left(\alpha(Y) \cap \pi^{-1}(\bigcup \mathscr{W})\right)=Y \cap \pi^{-1}(\bigcup \mathscr{W}) .
$$

We may assume that $\beta$ fixes $B_{1}^{*}$.

Construction of $\gamma$. We want $\gamma$ to move the set $Y^{\prime}=\bar{h}_{2} \circ \bar{h}_{1} \circ \beta \circ \alpha(Y)$ back to $\alpha(Y)$. Define the following covering of $\pi^{-1}(\bigcup \mathscr{W})$ :

(9) $\mathscr{W}^{\prime \prime}=\left\{\eta\left(W \times \mathbf{R}^{\infty} \times J\right): W \in \mathscr{W}, J\right.$ open $\subseteq[0,1]$ and $\left.\operatorname{diam} J<\varepsilon / 2\right\}$.

According to Lemma 3.3 the set $Y^{\prime}$ is an $\mathscr{M}_{k}^{\infty}$-absorber in $\mathbf{R}^{m} \times C$ so by Lemma 3.2 there exists a homeomorphism $\gamma$ of $\mathbf{R}^{m} \times C$ that is $\mathscr{W}^{\prime \prime}$-close to 1 with $\gamma\left(Y^{\prime}\right)=\alpha(Y)$ and $\gamma$ fixes $B_{1}^{*}$ (using that $Y^{\prime} \backslash \pi^{-1}(\bigcup \mathscr{W})$ is already equal to $\left.\alpha(Y) \backslash \pi^{-1}(\bigcup \mathscr{W})\right)$.

Define $g=\alpha^{-1} \circ \gamma \circ \bar{h}_{2} \circ \bar{h}_{1} \circ \beta \circ \alpha \mid Y$. Note that $g$ is a homeomorhism from $Y$ to $Y$ and that it is supported on $f^{-1}(\bigcup \mathscr{W})$. Observe that $Q=\overline{\cup \mathscr{W}}$ is disjoint from $A$ an hence $\tilde{g} \circ f_{A}=f_{A} \circ g$ defines a homeomorphism $\tilde{g}$ of $Y_{A}$. Since $\bigcup \mathscr{W} \subseteq U$ we have that $\tilde{g}$ fixes the complement of the set $\left(f^{A}\right)^{-1}(U)$. Recalling that $\beta, \bar{h}_{1}, \bar{h}_{2}$ and $\gamma$ all fix $B_{1}^{*}$, that $\alpha\left(K_{1}\right)=B_{1}^{*}$ and that $B \cap$ $\bigcup \mathscr{W} \subseteq B_{1}$ we find that $\tilde{g}$ fixes $K$.

Furthermore, if $y \in f^{-1}(\bigcup \mathscr{W})$ then

$$
f \circ g(y) \in \mathrm{St}^{3}(f(y), \mathscr{W})
$$


because $\pi \circ \beta, \pi \circ \bar{h}_{2}$ and $\pi \circ \gamma$ are $\mathscr{W}$-close to $\pi$ and $\pi \circ \alpha=\pi, \pi \circ \bar{h}_{2} \subseteq \pi$. In view of formula (4) we may conclude that $f \circ g$ and hence $f^{A} \circ \tilde{g}$ are $\mathscr{U}$-close to $f$ respectively $f^{A}$.

Now we verify condition (3) of Theorem 4.1 .

Case I. Let $b \in B \backslash B_{1}$ and select a $V \in \mathscr{V}_{1}$ that contains $b$. If we put $W=$ $V \cap O_{1}$ then $W$ is disjoint from $\bigcup \mathscr{W}$ and hence $g\left(f^{-1}(W)\right)=f^{-1}(W)$. Since $\pi^{-1}\left(\mathscr{V}_{1}\right)$ refines $f_{A}^{-1}(\mathscr{V})$ this takes care of the points of $B \backslash B_{1}$.

Case II. Let $b \in B_{1} \backslash B_{2}$ and select a $W \in \mathscr{W}_{1}$ that contains $b$. Using formula (10) and the fact that $\mathscr{W}$ refines $\mathscr{W}_{1}$ we find

$$
g\left(f^{-1}(W)\right) \subseteq f^{-1}\left(\operatorname{St}^{3}\left(W, \mathscr{W}_{1}\right)\right) .
$$

Formula (5) together with $\pi^{-1}\left(\mathscr{V}_{1}\right)$ refines $\pi_{A}^{-1}(\mathscr{V})$ allow us to conclude that $\tilde{g}\left(\left(f^{A}\right)^{-1}(W)\right) \subseteq V$ for some $V \in \mathscr{V}$.

Case III. Let $b \in B_{2}$ and select a $W \in \mathscr{W}^{\prime}$ that contains $b$. Note that $\alpha\left(f^{-1}(W)\right)=\pi^{-1}(W) \cap \alpha(Y)$ and (using $\operatorname{St}^{2}\left(B_{2}, \mathscr{W}^{\prime}\right) \subseteq P$ )

$$
\beta \circ \alpha\left(f^{-1}(W)\right) \subseteq f^{-1}\left(\operatorname{St}\left(W, \mathscr{W}^{\prime}\right)\right) \subseteq f^{-1}(P) .
$$

This leads to

$$
\bar{h}_{1} \circ \beta \circ \alpha\left(f^{-1}(W)\right) \subseteq f^{-1}\left(\mathbf{S t}^{2}(W, \mathscr{W})\right) \cap \bar{h}_{1}\left(f^{-1}(P)\right) .
$$

Using the fact that $\bar{h}_{2}$ pushes everything above the level $\delta$ inside the interval $(1-\varepsilon / 2,1]$ we find

$$
\bar{h}_{2} \circ \bar{h}_{1} \circ \beta \circ \alpha\left(f^{-1}(W)\right) \subseteq \eta\left(\mathrm{St}^{2}(W, \mathscr{W}) \times \mathbf{R}^{\infty} \times(1-\varepsilon / 2,1]\right) .
$$

As a consequence of formulae (9) and (4) we find

(15) $\gamma \circ \bar{h}_{2} \circ \bar{h}_{1} \circ \beta \circ \alpha\left(f^{-1}(W)\right) \subseteq \eta\left(\mathrm{St}^{3}(W, \mathscr{W}) \times \mathbf{R}^{\infty} \times(1-\varepsilon, 1]\right) \subseteq \alpha\left(\pi_{A}^{-1}(V)\right)$

for some $V$ in $\mathscr{V}$. So we may conclude that $g\left(f^{-1}(W)\right) \subseteq f_{A}^{-1}(V)$ and hence $\bar{g}\left(\left(f^{A}\right)^{-1}(W)\right) \subseteq V$.

Case IV. The case $b \in Y_{B} \backslash B$ is a triviality since $f_{B}^{A} \mid\left(f_{B}^{A}\right)^{-1}\left(Y_{B} \backslash B\right)$ is the identity homeomorphism on $((X \backslash B) \times C) \cap Y$.

Now we are ready for

Proof of Proposition 4.3. We want to show that $f: Y \rightarrow X$ is a near homeomorphism. Let $d$ be an arbitrary metric on $X$. Write $X=\bigcup_{i=0}^{\infty} X_{i}$ and $Y=\bigcup_{i=0}^{\infty} Y_{i}$, where the $X_{i}$ 's and $Y_{i}$ 's are compacta such that $X_{i} \subseteq X_{i+1}$ and $Y_{i} \subseteq Y_{i+1}$. We may choose $X_{0}$ and $Y_{0}$ to be empty. Put $A_{0}=\varnothing, g_{0}=1_{Y}$ : $Y \rightarrow Y_{A_{0}}=Y$ and let $\mathscr{U}_{0}$ be an arbitrary open covering of $X$ with mesh $<1 / 2$. This is the base step of an induction process in which we shall construct a sequence of compacta $A_{0} \subseteq A_{1} \subseteq A_{2} \subseteq \cdots \subseteq X$, a sequence of homeomorphisms $g_{i}: Y_{A_{i-1}} \rightarrow Y_{A_{i}}$ and a sequence of normal covers $\mathscr{U}_{i}$ of $X \backslash A_{i}$ rel $A_{i}$ such that

(1) $\operatorname{mesh}\left(\mathscr{U}_{i}\right)<2^{-i-1}$,

(2) $\mathscr{U}_{i+1}$ is a star refinement of $\mathscr{U}_{i}$,

(3) $h_{i}$ and $h_{i+1}$ are $\mathscr{U}_{i}$-close,

(4) $X_{i} \cup h_{i}\left(Y_{i}\right) \subseteq A_{i}$,

where $h_{i}: Y \rightarrow X$ denotes $f^{A_{i}} \circ g_{i} \circ \cdots \circ g_{1} \circ g_{0}$. 
First, let us assume that the induction is performed and show that $h=$ $\lim _{i \rightarrow \infty} h_{i}$ is a homeomorphism that approximates $f$. If $y \in Y$ then $y \in$ $Y_{i}$ for some $i$ so $h_{i}(y) \in A_{i}$. Since $\bigcup \mathscr{U}_{i}=X \backslash A_{i}$ we have that $h_{i}(y)=$ $h_{i+1}(y)=h_{i+2}(y)=\cdots$. So $h$ exists and is a surjection by formula (4). Since $\operatorname{mesh}\left(\mathscr{U}_{i}\right)<2^{-i-1}$ we have $h$ is continuous and $d(h, f)<1$. For the final step let $U$ be a neighbourhood of $y \in Y$ and let $i$ be such that $y \in Y_{i}$. Since $h_{i}(y) \in A_{i}$ and by the definition of $h_{i}$ we have that $y$ is the only point to be mapped onto $h_{i}(y)$ by $h_{i}$. Note that $f^{A_{i}}$ and hence $h_{i}$ are closed over $A_{i}$. This means that there is a neighbourhood $V$ of $h_{i}(y)$ in $X$ such that $h_{i}^{-1}(V) \subseteq U$. Since $\mathscr{U}_{i}$ is normal $\operatorname{rel} A_{i}$ there is a neighbourhood $W$ of $h_{i}(y)$ in $X$ such that $\operatorname{St}^{2}\left(W, \mathscr{U}_{i}\right) \subseteq V$. Let $z$ be an element of $h^{-1}(W)$, which is a neighbourhood of $y$ because $h_{i}(y)=h(y)$. Let $z \in Y_{j}$ for some $j>i$. Then we have $h_{j}(z)=h(z) \in W$. This implies that $h_{i}(z)$ is an element of

$$
\operatorname{St}\left(\cdots \operatorname{St}\left(\operatorname{St}\left(W, \mathscr{U}_{j-1}\right), \mathscr{U}_{j-2}\right) \cdots, \mathscr{U}_{i}\right) .
$$

Since $\mathscr{U}_{k+1}$ is a star refinement of $\mathscr{U}_{k}$ we find that the set in (5) is contained in $\operatorname{St}^{2}\left(W, \mathscr{U}_{i}\right)$ which in turn is a subset of $V$. So $h_{j}(z) \in V$ and hence $z \in h_{j}^{-1}(v) \subseteq U$. Since we found that $h^{-1}(W) \subseteq U$ we may conclude that $h$ is a homeomorphism.

Assume that we have constructed $A_{i}, g_{i}$ and $\mathscr{U}_{i}$. Let $\alpha=g_{i} \circ \cdots g_{1} \circ g_{0}$ : $Y \rightarrow Y_{A_{i}}$ and put

$$
K_{1}=\alpha\left(Y_{i+1}\right) \cup A_{i} \subseteq Y_{A_{i}} .
$$

Extend the elements of $\mathscr{U}_{i}$ to open subsets of $\mathbf{R}^{m}$. Let $\mathscr{U}^{\prime}$ be an open cover of $\bigcup \mathscr{U}_{i}$ such that $\mathrm{St}^{2}\left(\mathscr{U}^{\prime}\right)$ refines $\mathscr{U}_{i}$. According to the Corollaries 4.5 and 3.6 we can find an open cover $\mathscr{V}$ of $Y_{A_{i}} \backslash A_{i}=\left(\left(X \backslash A_{i}\right) \times C\right) \cap Y$ in $\mathbf{R}^{m} \times C$ such that

$$
\mathscr{V} \text { refines }\left(\pi^{A_{i}}\right)^{-1}\left(\mathscr{U}^{\prime}\right)
$$

and whenever $\alpha_{1}$ and $\alpha_{2}$ are two imbeddings of a compact space into $Y_{A_{i}}$ that are $\operatorname{St}(\mathscr{V})$-close then there is a homeomorphism $\beta$ of $Y_{A_{i}}$ such that $\beta$ is $\left(f^{A_{i}}\right)^{-1}\left(\mathscr{U}^{\prime}\right)$-close to 1 and $\beta \circ \alpha_{1}=\alpha_{2}$.

Let $\mathscr{V}^{\prime}$ be an open covering of $f^{A_{i}}\left(K_{1} \backslash A_{i}\right)$ in $\bigcup \mathscr{U}_{i}$ such that $\mathscr{V}^{\prime}$ refines $\mathscr{U}^{\prime}$ and for every $V \in \mathscr{V}^{\prime}$ there is a collection $\mathscr{O}$ of open subsets of $C$ such that

$$
\begin{aligned}
& \{V \times O: O \in \mathscr{O}\} \quad \text { refines } \mathscr{V} \text { and } \\
& (V \times \bigcup \mathscr{O}) \cap K_{1}=(V \times C) \cap K_{1} .
\end{aligned}
$$

Let $\phi$ be an imbedding of $K_{1} \rightarrow X$ that is $\mathscr{V}^{\prime}$-close to $f^{A_{i}} \mid K_{1},[11, \S 4.4]$, Lemma 2.2 and Definition 2.3. Put $B=\phi\left(K_{1}\right)$ and

$$
A_{i+1}=X_{i+1} \cup \phi\left(K_{1}\right) \text {. }
$$

Let $\psi=\pi^{\prime} \circ \phi^{\prime} \mid\left(B \backslash A_{i}\right):\left(B \backslash A_{i}\right) \rightarrow C$, where $\pi^{\prime}$ stands for the projection $\mathbf{R}^{m} \times C \rightarrow \mathbf{R}^{m}$, and let $\bar{\psi}$ be an extension of $\psi$ over $A_{i+1} \backslash A_{i}$. Define the compact set $K$ in $\left(\mathbf{R}^{m} \times C\right)_{A_{i}}$ by

$$
K=A_{i} \cup\left\{(b, \bar{\psi}(b)): b \in A_{i+1} \backslash A_{i}\right\} .
$$


Observe that $\pi^{A_{i}} \mid K: K \rightarrow A_{i+1}$ is a homeomorphism. Let the imbedding $\chi: K_{1} \rightarrow K$ be given by

$$
\begin{cases}\chi(a)=a & \text { if } a \in A_{i}, \\ \chi(x, y)=(\phi(x, y), y) & \text { if }(x, y) \in K_{1} \backslash A_{i} .\end{cases}
$$

The continuity of $\chi$ at points of $A_{i}$ follows from the fact that $\phi$ is $\mathscr{U}_{i}$-close to $f^{A_{i}} \mid K_{1}$ and that $\mathscr{U}_{i}$ is normal rel $A_{i}$ One may verify that (8) and (9) guarantee that $\chi$ is $\mathscr{V}$-close to $1_{K_{1}}$.

Since $K \backslash A_{i}$ is a $\leq k$-dimensional $\sigma$-compactum in $\left(\mathbf{R}^{m} \backslash A_{i}\right) \times C$ we have that

$$
Y^{\prime}=Y \cup\left(K \backslash A_{i}\right)
$$

is an $\mathscr{M}_{k}^{\infty}$-absorber in $\mathbf{R}^{m} \times C$. Define the following covering of $\pi^{-1}\left(\bigcup \mathscr{U}_{i}\right)$ :

$$
\mathscr{W}=\mathscr{V} \cup\left\{\pi^{-1}(U) \backslash \chi\left(K_{1}\right): U \in \mathscr{U}^{\prime}\right\} .
$$

By Lemma 3.2 there is a homeomorphism $\gamma$ of $\mathbf{R}^{m} \times C$ that is $\mathscr{W}$-close to 1 with the property $\gamma(Y)=Y^{\prime}$. Since $\gamma$ is $\pi^{-1}\left(\mathscr{U}_{i}\right)$-close to 1 and since $\mathscr{U}_{i}$ is normal rel $A_{i}$ we find that $\tilde{\gamma} \circ \pi_{A_{i}}=\pi_{A_{i}} \circ \gamma$ defines a homeomorphism $\tilde{\gamma}$ of $\left(\mathbf{R}^{m} \times C\right)_{A_{i}}$. Note that $\tilde{\gamma} \mid Y_{A_{i}}: Y_{A_{i}} \rightarrow Y_{A_{i}}^{\prime}$ is also a homeomorphism.

It is easily verified that $1_{K_{1}}$ is $\operatorname{St}(\mathscr{V})$-close to $\tilde{\gamma} \circ \chi: K_{1} \rightarrow Y_{A_{i}}$. So there is a homeomorphism $\beta$ of $Y_{A_{i}}$ such that $f^{A_{i}} \circ \beta$ and $f^{A_{i}}$ are $\mathscr{U}^{\prime}$-close and

$$
\beta \mid K_{1}=\tilde{\gamma}^{-1} \circ \chi .
$$

We are now ready to apply Lemma 4.4 to

$$
f_{A_{i+1}}^{\prime A_{i}}: Y_{A_{i}}^{\prime} \rightarrow Y_{A_{i+1}}^{\prime} \text {. }
$$

Note that $Y^{\prime}$ meets the requirements of Lemma 4.4 because $Y \subseteq Y^{\prime}, \pi\left(Y^{\prime}\right)=$ $X$ and $Y^{\prime}$ "projects" onto an $\mathscr{M}_{k}$-absorber $X_{1}^{\prime}$ in $\mathbf{R}^{m} \times \mathbf{R}^{\infty}$. We observed earlier that $\pi^{A_{i}} \mid K$ is a homeomorphism between $K$ and $A_{i+1}$. So there exists a homeomorphism $H: Y_{A_{i}}^{\prime} \rightarrow Y_{A_{i+1}}^{\prime}$ that is $\left(\pi^{A_{i+1}}\right)^{-1}\left(\mathscr{U}^{\prime}\right)$-close to $f_{A_{i+1}}^{\prime A_{i}}$ and that has the property

$$
H\left|K=f_{A_{i+1}}^{\prime A_{i}}\right| K
$$

Since $K \subseteq\left(\pi^{A_{i}}\right)^{-1}\left(A_{i+1}\right)$ we have that $Y_{A_{i+1}}$ is equal to $Y_{A_{i+1}}^{\prime}$. Observe that

$$
g_{i+1}=H \circ \tilde{\gamma} \circ \beta
$$

is a homeomorhism from $Y_{A_{i}}$ onto $Y_{A_{i+1}}$ with the property

$$
\begin{aligned}
f^{A_{i+1}} \circ g_{i+1}\left(K_{1}\right) & =f^{A_{i+1}} \circ H \circ \tilde{\gamma} \circ \beta\left(K_{1}\right) \\
& =f^{A_{i+1}} \circ H \circ \chi\left(K_{1}\right) \\
& \subseteq f^{A_{i+1}} \circ H(K)=f^{\prime A_{i}}(K)=A_{i+1} .
\end{aligned}
$$

Consequently,

$$
\begin{aligned}
h_{i+1}\left(Y_{i+1}\right) & =f^{A_{i+1}} \circ g_{i+1} \circ \alpha\left(Y_{i+1}\right) \\
& \subseteq f^{A_{i+1}} \circ g_{i+1}\left(K_{1}\right) \subseteq A_{i+1} .
\end{aligned}
$$

This and (10) take care of induction hypothesis (4). The maps $\pi^{A_{i}} \circ \beta, \pi^{A_{i}} \circ \gamma$ and $\pi^{A_{i+1}} \circ H$ are all $\mathscr{U}^{\prime}$-close to $\pi^{A_{i}}$ so $f^{A_{i+1}} \circ g_{i+1}$ is $\operatorname{St}^{2}\left(\mathscr{U}^{\prime}\right)$-close to $f^{A_{i}}$ and hence $h_{i+1}$ is $\mathscr{U}_{i}$-close to $h_{i}$, hypothesis (3). 
Finally, let $\mathscr{U}_{i+1}$ be a normal cover of $X \backslash A_{i+1} \operatorname{rel} A_{i+1}$ with mesh less than $2^{-i-2}$ and which moreover is a star refinement of $\mathscr{U}_{i}$.

Proposition 4.3 together with the uniqueness of absorbers gives

4.6. Theorem. Let $n \geq 2 k+1$ be integers and let $B_{k}^{n}$ and $B_{k}^{\infty}$ be the universal $k$-dimensional pseudo-boundaries in $\mathbf{R}^{n}$ and $\mathbf{R}^{\infty}$, respectively. Then $B_{k}^{n}$ and $B_{k}^{\infty}$ are homeomorphic.

4.7. Theorem. Let $n, m \geq 2 k+1$ be integers and let $s_{k}^{n}$ and $s_{k}^{m}$ be the universal $k$-dimensional pseudo-interiors in $\mathbf{R}^{n}$ and $\mathbf{R}^{m}$, respectively. Then $s_{k}^{n}$ and $s_{k}^{m}$ are homeomorphic.

Proof. By Lemma 2.5 there are an $\mathscr{M}_{k}^{n}$-absorber $X$ in $\mathbf{R}^{n}$ and an $\mathscr{M}_{k}^{m}-$ absorber $Y$ in $\mathbf{R}^{m}$ such that $X \subseteq s_{k}^{n}$ and $Y \subseteq s_{k}^{m}$. By Theorem 4.6 and [16] there exists a homeomorphism $h: X \rightarrow Y$. By Lavrentiev's Theorem [2, p. 21] $h$ can be extended to a homeomorphism $\bar{h}: \vec{X} \rightarrow \vec{Y}$ between certain $G_{\delta}$-sets, $X \subseteq \vec{X} \subseteq s_{k}^{n}, Y \subseteq \vec{Y} \subseteq s_{k}^{m}$. By Lemma 2.6, $\mathbf{R}^{n} \backslash \vec{X} \in \sigma \mathscr{M}_{n-k-1}^{n}$ and $\mathbf{R}^{m} \backslash \vec{Y} \in \sigma \mathscr{M}_{m-k-1}^{m}$. By Lemma 2.4, $s_{k}^{n}$ and $\vec{X}$ are homeomorphic and $s_{k}^{m}$ and $\vec{Y}$ are homeomorphic. We conclude that $s_{k}^{n}$ and $s_{k}^{m}$ are homeomorphic.

4.8. Corollary. All imbeddings of a compactum in $s_{k}^{2 k+1}$ or $B_{k}^{2 k+1}$ are equivalent, i.e., every homeomorphism between compacta in $s_{k}^{2 k+1}$ or $B_{k}^{2 k+1}$ can be extended to a homeomorphism of $s_{k}^{2 k+1}$ respectively $B_{k}^{2 k+1}$.

For instance, the trefoil and the unknot are topologically indistinguishable in $s_{1}^{3}$ or $B_{1}^{3}$. Corollary 4.8 is known for $n \geq 2 k+2$. This follows from the unknotting theorem in $\mathbf{R}^{n}$ for elements of $\mathscr{M}_{k}^{n}$ (Geoghegan and Summerhill [9, Theorem 2.5]) and Lemma 2.4 (cf. Dijkstra [6, Theorem 1.2.13]).

We conclude this section with two conjectures. The stable pseudo-interiors $s_{k}^{n}, n>2 k$, behave like $k$-dimensional versions of Hilbert space $l^{2}$. It is expected that this analogy extends to Torunczyk's [14] characterization of $l^{2}$ :

4.9. Conjecture. The space $s_{k}^{n}$ for $n>2 k$ is characterized topologically by the following properties:

(1) $k$-dimensionality,

(2) topological completeness,

(3) $L C^{k-1}$ and $C^{k-1}$,

(4) the discrete $k$-cells property.

A space $X$ has the discrete $k$-cells property if every sequence $\left(f_{i}: I^{k} \rightarrow X\right)_{i=1}^{\infty}$ can be approximated by a sequence $\left(g_{i}: I^{k} \rightarrow X\right)_{i=1}^{\infty}$ such that the images of the $g_{i}$ 's form a discrete collection in $X$.

The stable pseudo-boundaries $B_{k}^{n}$ for $n>2 k$ are $k$-dimensional versions of the pseudo-boundary $B$ of the Hilbert cube, which space was characterized by Mogilski [12]. This leads to

4.10. Conjecture. The space $B_{k}^{n}$ for $n>2 k$ is characterized topologically by the following properties:

(1) $k$-dimensionality, 
(2) $\sigma$-compactness,

(3) $L C^{k-1}$ and $C^{k-1}$,

(4) the discrete $k$-cells property,

(5) strong universality for $\leq k$-dimensional compacta.

If $\mathscr{M}$ is a collection of spaces then a space $X$ is called strongly $\mathscr{M}$-universal if every map $f$ from an element $C$ of $\mathscr{M}$ into $X$ such that $f \mid D: D \rightarrow X$ is a $Z$-imbedding for some closed subset $D$ of $C$, can be approximated by a $Z$-imbedding $g: C \rightarrow X$ that restricts to $f$ on $D$.

\section{Negative Results}

We shall now prove that the spaces $B_{k}^{n}$ and $B_{k}^{m}$ and the spaces $s_{k}^{n}$ and $s_{k}^{m}$ are not homeomorphic if $n \leq 2 k$.

We say that a space has property $D(i, j)$ if for every pair of maps $f: I^{i} \rightarrow X$ and $g: I^{j} \rightarrow X$ there exists maps $f^{\prime}: I^{i} \rightarrow X$ and $g^{\prime}: I^{j} \rightarrow X$ arbitrarily close to $f$ and $g$, respectively, with $f^{\prime}\left(I^{i}\right) \cap g^{\prime}\left(I^{j}\right)=\varnothing$.

The following lemma is probably well known. We include a proof for completeness sake.

\subsection{Lemma. $\mathbf{R}^{i+j+1}$ has $D(i, j)$ whereas $\mathbf{R}^{i+j}$ has not.}

Proof. That $\mathbf{R}^{i+j+1}$ has $D(i, j)$ is easy: simply approximate $f$ and $g$ by $P L$-maps and bring their images into general position.

We proceed to prove that $\mathbf{R}^{i+j}$ does not have $D(i, j)$. Let $J=[-1,1]$ and let $f: J^{i} \rightarrow \mathbf{R}^{i+j}$ and $g: J^{j} \rightarrow \mathbf{R}^{i+j}$ be given by $f(x)=(x, 0)$ and $g(y)=$ $(0, y)$. Let $f^{\prime}$ and $g^{\prime}$ be two functions with disjoint images that are close to $f$ and $g$, respectively. Define $\alpha: J^{i+j} \rightarrow \mathbf{R}^{i+j} \backslash\{0\}$ by $\alpha(x, y)=f^{\prime}(x)-g^{\prime}(-y)$. Note that $\alpha$ is close to the identity and hence $\alpha \mid \partial J^{i+j}$ is an essential map into $\mathbf{R}^{i+j} \backslash\{0\}$. Consequently, the extension over $J^{i+j}$ cannot exist.

5.2. Proposition. $B_{k}^{n}$ and $s_{k}^{n}$ have the property $D(k, \min \{k, n-k-1\})$. Moreover, if $n \leq 2 k$ then $B_{k}^{n}$ and $s_{k}^{n}$ do not have $D(k, n-k)$.

Proof. Consider $B_{k}^{n}, \varepsilon>0, f: I^{k} \rightarrow B_{k}^{n}$ and $g: I^{m} \rightarrow B_{k}^{n}$, where $m=$ $\min \{k, n-k-1\}$. There are $f_{1}: I^{k} \rightarrow \mathbf{R}^{n}$ and $g_{1}: I^{m} \rightarrow \mathbf{R}^{n}, \frac{\varepsilon}{3}$-close to $f$ and $g$, and with disjoint images. Find $P L$-approximations $f_{2}$ and $g_{2}$ to $f_{1}$ and $g_{1}$ that are

$$
\min \left\{\frac{\varepsilon}{3}, \frac{d\left(f_{1}\left(I^{k}\right), g_{1}\left(I^{m}\right)\right)}{2}\right\}
$$

close. The polyhedra $f_{2}\left(I^{k}\right)$ and $g_{2}\left(I^{m}\right)$ are disjoint and at most $k$-dimensional. So $f_{2}\left(I^{k}\right) \cup g_{2}\left(I^{m}\right)$ is an element of $\mathscr{M}_{k}^{n}$ and can be pushed into the absorber $B_{k}^{n}$ by a homeomorphism $\alpha$ with distance less than $\frac{\varepsilon}{3}$ towards the identity. Consequently, $\alpha \circ f_{2}$ and $\alpha \circ g_{2}$ are $\varepsilon$-close to $f$ and $g$ and have disjoint images.

For $s_{k}^{n}$ the argument is similar with one exception: $f_{2}\left(I^{k}\right)$ and $g_{2}\left(I^{m}\right)$ are elements of $\mathscr{M}_{k}^{n}$ and can be pushed off the $\mathscr{M}_{n-k-1}^{n}$-absorber $B_{n-k-1}^{n}$ and thus into $s_{k}^{n}$ (Lemma 2.5).

Consider now the case $n \leq 2 k$. Let $f: I^{k} \rightarrow \mathbf{R}^{n}$ and $g: I^{n-k} \rightarrow \mathbf{R}^{n}$ be two maps that do not have $\varepsilon$-approximations by functions with disjoint images (Lemma 5.1). We may assume that $f$ and $g$ are piecewise linear. As above 
we can find maps $f_{1}$ and $g_{1}$ from $I^{n}$ and $I^{n-k}$ into $B_{k}^{n}$ (or $s_{k}^{n}$ ) that have distance less than $\frac{\varepsilon}{2}$ towards $f$ and $g$. Then every pair $f^{\prime}$ and $g^{\prime}$ with distance less than $\frac{\varepsilon}{2}$ towards $f_{1}$ and $g_{1}$ has intersecting images.

\section{REFERENCES}

1. R. D. Anderson, Strongly negligible sets in Fréchet manifolds, Bull. Amer. Math. Soc. 75 (1969), 64-67.

2. C. Bessaga and A. Pelczyński, Selected topics in infinite-dimensional topology, PWN, Warsaw, 1975.

3. M. Bestvina and J. Mogilski, Characterizing certain incomplete infinite-dimensional absolute retracts, Michigan Math. J. 33 (1986), 291-313.

4. T. A. Chapman, Dense sigma-compact subsets of infinite-dimensional manifolds, Trans. Amer. Math. Soc. 154 (1971), 399-426.

5. D. W. Curtis and J. van Mill, Zero-dimensional countable dense unions of Z-sets in the Hilbert cube, Fund. Math. 118 (1983), 103-108.

6. J. J. Dijkstra, Fake topological Hilbert spaces and characterizations of dimension in terms of negligibility, CWI Tract 2, Amsterdam, 1984.

7. $\ldots$, k-dimensional skeletoids in $\mathbf{R}^{n}$ and the Hilbert cube, Topology Appl. 19 (1985), 13-28.

8. F. van Engelen and J. van Mill, Borel sets in compact spaces: some Hurewicz-type theorems, Fund. Math. 124 (1984), 271-286.

9. R. Geoghegan and R. R. Summerhill, Concerning the shapes of finite-dimensional compacta, Trans. Amer. Math. Soc. 179 (1973), 281-292.

10. _ Pseudo-boundaries and pseudo-interiors in euclidean spaces and topological manifolds, Trans. Amer. Math. Soc. 194 (1974), 141-165.

11. J. van Mill, Infinite-dimensional topology: Prerequisites and introduction, North-Holland, Amsterdam, 1989.

12. J. Mogilski, Characterizing the topology of infinite-dimensional $\sigma$-compact manifolds, Proc. Amer. Math. Soc. 92 (1984), 111-118.

13. H. Torunczyk, Concerning locally negligible sets and characterization of $l_{2}$-manifolds, Fund. Math. 101 (1978), 93-110.

14. __ A correction of two papers concerning Hilbert manifolds, Fund. Math. 125 (1985), 89-93.

15. __ Skeletons and absorbing sets in complete metric spaces, Doctoral thesis, Institute of Mathematics of the Polish Academy of Sciences; unpublished.

16. J. E. West, The ambient homeomorphy of an incomplete subspace of an infinite-dimensional Hilbert space, Pacific J. Math. 34 (1970), 247-267.

17. R. Y. T. Wong, A wild Cantor set in the Hilbert cube, Pacific J. Math. 24 (1968), 189-193.

Department of Mathematics, University of Alabama, Box 870350, Tuscaloosa, AlABAMA 35487-0350

E-mail address: jdijkstr@ualvm

Faculteit Wiskunde en Informatica, Vrije Universiteit, Postbus 7161, 1007 MC AmsTERDAM, THE NETHERLANDS

E-mail address: vanmill@cs.vu.nl

Instytut Matematyki, Uniwersytet Warszawski, PKiN, 00-901 Warszawa, Poland

Current address: Department of Mathematics and Statistics, Southern Illinois University, Edwardsville, Illinois 62026

E-mail address: yd00@siuemus 